\title{
Magnitude and variation of the critical power law exponent and its physical controls
}

\author{
Sunji Zhou ${ }^{a}$, Shengwang Hao ${ }^{a, b, *}$, Derek Elsworth ${ }^{c}$ \\ ${ }^{\text {a }}$ School of Civil Engineering and Mechanics, Yanshan University, Qinhuangdao, China \\ ${ }^{\mathrm{b}}$ The State Key Laboratory of Nonlinear Mechanics, Institute of Mechanics, Chinese Academy of Science, Beijing, China \\ ${ }^{\mathrm{c}}$ Energy and Mineral Engineering, Geosciences, $G^{3}$ Center, and EMS Energy Institute, Pennsylvania State University, University \\ Park, PA, USA
}

\section{H I G H L I G H T S}

- Physical mechanism of variation of the critical power law precursor exponent.

- Range of the critical power law exponent.

- Relationship of critical exponent with the degree of local stress controlling damage.

\section{A R T I C L E I N F O}

\section{Article history:}

Received 17 March 2018

Received in revised form 23 June 2018

Available online 19 July 2018

\section{Keywords:}

Critical power law precursor

Failure

Exponent

Magnitude

\begin{abstract}
A B S T R A C T
We study the physical controls on the scatter of exponents in the critical power law relation that describes an acceleration in precursory signals of deformation (displacements) or seismicity (damage) in the vicinity of failure time. Based on the time-dependent fiber bundle model and equal load share (ELS) rule, we find that the critical power law exponents range from -0.5 to -1.0 . And values of the critical power law exponents depends on a parameter $\rho$, which defines the sensitivity of damage growth in a fiber to the local stress. Both the simulation results and theoretical analysis demonstrate that the critical power law precursor exponent $-\beta$ has a relationship $-\beta=-(1-1 / \rho)$ with $\rho$. Thus, our results illustrate a physical mechanism of variation of the critical power law exponent that is determined by the degree of the local stress controlling the damage evolution of a fiber.
\end{abstract}

(c) 2018 Elsevier B.V. All rights reserved.

\section{Introduction}

Monitoring the critical acceleration of measurable quantities such as displacements or (micro-)seismicity is widely accepted as a proxy for a precursor for failure and for the prediction of time-to-failure [1-8]. This is often analyzed as a critical phenomenon [9-12] with a power-law divergence of physical quantities at failure time $t_{f}$. In the vicinity of the failure time, the critical acceleration exhibited by a measurable quantity can be described as a power law relation $[1-3,7,8,13-15]$ with respect to the time to failure

$$
\dot{\Omega}=k\left(t_{f}-t\right)^{-\beta} \text {. }
$$

where $\Omega$ represents a measurable quantity and the overscripted dot represents the derivative of $\Omega$ with respect to time. $-\beta$ is the critical power law exponent. Eq. (1) can be deduced from the Voight relation $[1,2,7,8,13]$

$$
\dot{\Omega}^{-\alpha} \ddot{\Omega}-A=0
$$

\footnotetext{
* Corresponding author at: School of Civil Engineering and Mechanics, Yanshan University, Qinhuangdao, China.

E-mail address: hsw@ysu.edu.cn (S. Hao).
} 
that describes the behavior of materials in their terminal stage of failure, with $k=[A(\alpha-1)]^{1 /(1-\alpha)}$ and $\beta=1 /(\alpha-1)^{[1]}$. Where $A$ and $\alpha$ are constants recovered from curve-fitting of observations.

Eq. (1) can be rewritten in a linearized form [14]

$$
\dot{\Omega}^{-1 / \beta}=k^{-1 / \beta}\left(t_{f}-t\right) .
$$

Then the failure time can be determined by linearly extrapolating the curve of $\dot{\Omega}^{-1 / \beta}$ versus time to its intersection with the time axis. This application of Voight's relation is known as the Failure Forecast Method (FFM) [14-17]. This method has been demonstrated to work well in the retrospective prediction of laboratory experiments [18,19], landslides [20,21], and volcanic eruptions [2-5,22]. In application of the FFM, the linearization of the power law trend is usually used to predict the failure time. This leads to a biased and imprecise prediction compared to a fully nonlinear fitting method because the error structure is no longer Gaussian after the transformation of the rate data [23].

However, the uncertainty resulting from the scatter of the critical exponent $-\beta$ is a key difficulty in using such method [24]. For example, Voight [1] discovered that $-\beta$ is approximately -1.0 for soils. For creep-relaxation experiments on rock, Hao et al. [25] found that the exponent $-\beta$ is approximately $-2 / 3$. Smith and Kilburn [22] found that $-\beta$ took values of approximately -0.43 for 3 data points for the 1991 Mount Pinatubo eruption (Philippines) and tend to -0.91 for 7 data points. Analysis [8,26-28] based on fiber bundle models and global mean-field approximations demonstrated that $-\beta=-0.5$. It has also been observed that precursory signals may evolve with time from conditions for $\alpha \approx 1$ to conditions for $\alpha \approx 2(-\beta=-1.0)$ [5,6,20]. Thus, it is particularly important to understand the underlying mechanisms for the magnitude and variation of critical power-law exponents.

In materials science and engineering, the fiber bundle model has been widely used [29-31] to analyze the failure properties of heterogeneous materials. A time-dependent implementation of this method was first proposed by Coleman [31], representing the response of fiber bundles where each fiber has an independent lifetime drawn from some distribution. According to the load sharing rules, the fiber bundle models can be divided into two extreme types: equal load sharing (ELS) and the local load sharing (LLS) models [32-34].

The local load-sharing (LLS) fiber bundle model was introduced by Harlow and Phoenix [32,33]. In this model, when a fiber fails, the load it carried is redistributed equally onto its two nearest surviving neighbors. Hence, a survival fiber carries the load

$$
\sigma=K \sigma_{0},
$$

with the stress concentration factor

$$
K=1+(l+r) / 2 .
$$

where $l$ and $r$ represent the number of broken fibers on the left or right of this survival fiber respectively. $\sigma_{0}$ is the load on every fiber at the initial state when all fibers are intact.

In the ELS model, the load is shared equally by all surviving elements in the system. Thus if $N_{\mathrm{b}}$ fibers have broken, every surviving fiber has the same stress concentration factor

$$
K=\frac{N}{N-N_{b}(t)}=\frac{1}{1-N_{b} / N}
$$

and carries the same stress $\sigma=K \sigma_{0} . N_{b}(t)$ is the number of broken fibers up to time $t$. ELS model has a merit to give a theoretical analysis of continuous cases because some closed form analytic results can be obtained.

In this paper, we perform an analytical derivation about the critical power law precursor based on the ELS rule. We illustrate that the critical power law exponents change from -0.5 to -1.0 . The relationship between the critical exponent with the parameter that reflects the nonlinear relationship between damage rate of a fiber and the local stress, is derived. Then, Monte Carlo simulations are performed to explicitly show the magnitude and variation of the critical exponent.

\section{Bundle geometry and stochastic fiber lifetime model}

We consider a fiber bundle consisting of $N$ fibers that are arranged around the circumference of a circle, with each of them with two adjacent neighbors. We apply a fixed load $N \sigma_{0}$ to the bundle; that is, initially each fiber is intact and carries load $\sigma_{0}$. As time progresses, fibers break and thus surviving fibers share load according to the desired load redistribution rule.

When a fiber is subjected to a load history $\sigma(t), t>0$, its consumed lifetime during this loading process is described by [31,34]

$$
T(t)=\int_{0}^{t} \kappa(\sigma(s)) d s
$$

where $\kappa(\sigma)$ is usually referred to as the breakdown rule [30,33], and is usually described by a power law relation [34-36]

$$
\kappa(\sigma)=\kappa_{0}\left(\frac{\sigma}{\eta}\right)^{\rho} .
$$


where $\kappa_{0}$ and $\eta$ are constants. $\sigma$ is the local stress on a fiber and $\rho$ is the parameter that accounts for the sensitivity of damage growth of a fiber on the local stress $\sigma . \rho$ must be greater than 1 to have a non-linear, accelerating relation of break rate.

Every fiber is assumed to have a specific lifetime $T_{\mathrm{s}}$, and follows the Weibull distribution, i.e.

$$
P\left(T_{s}\right)=1-\exp \left[-\Psi\left(T_{s}\right)\right] .
$$

where $\Psi(x)(x \geq 0)$ is the hazard function that can be expressed as

$$
\Psi(x)=x^{\theta} .
$$

and $\theta$ is the Weibull parameter. Models [8,26-28] where the effects of the load history on the consumption of lifetime are neglected result in the critical power law exponent $-\beta=-0.5$. In these models, the breaking of every fiber element is totally determined by its specific strength threshold. Thus, these cannot describe the nonlinear dependence of lifetime of each fiber on the history of local stress.

\section{Critical scaling law of break-rate with time to failure under ELS}

\subsection{Analytical results under ELS}

In order to demonstrate the critical power law behavior of break rate and the variation of the critical exponents, we perform an analytical derivation based on a continuous case under ELS.

Based on Eqs. (7) and (9), the broken fraction, i.e. $P=N_{b} / N$ the ratio of the number $N_{b}(t)$ of broken fibers with respect to the total number $N$ of fibers is

$$
P(t)=1-\exp \left[-\left(\int_{0}^{t} \kappa(\sigma(s)) d s\right)^{\theta}\right] .
$$

From Eq. (6), the true load on every surviving fiber is

$$
\sigma(t)=\frac{\sigma_{0}}{1-P(t)}
$$

Then, the break rate can be expressed as

$$
\frac{1}{N} \frac{d N_{b}}{d t}=\frac{d P}{d t}=\exp \left[-\left(\int_{0}^{t} \kappa(\sigma(s)) d s\right)^{\theta}\right] \theta\left(\int_{0}^{t} \kappa(\sigma(s)) d s\right)^{\theta-1} \kappa(\sigma(t)) .
$$

Substituting Eq.(8) into (13) gives

$$
\frac{d P}{d t}=\theta\left(\int_{0}^{t} \kappa(\sigma(s)) d s\right)^{\theta-1} \kappa_{0}\left(\frac{\sigma_{0}}{\eta}\right)^{\rho}\left(\frac{1}{1-P}\right)^{\rho-1} .
$$

Consequently, when $\rho=2,3,4, \ldots \ldots$, that the bundle approaches the complete failure, i.e. $P \rightarrow 1$ leads to $\frac{d P}{d t} \rightarrow \infty$ and $\frac{d t}{d P} \rightarrow 0$. Further, Eq. (14) indicates that the $\lambda<\rho$ order derivatives of $t$ with respect to $P, \frac{d^{\lambda} t}{d P^{\lambda}}$ is a function of $(1-P)^{\rho-\lambda}$. Noting that, at the point of complete failure, $P=1$ leads to the requirement that $\frac{d^{\lambda} t}{d P^{\lambda}}$ is equal to zero.

To clearly demonstrate the critical power law behavior of break rate, we turn to the series expansion of $t(P)$ as a function of $P$ in the vicinity of failure time $t_{f}$ and omit the terms higher than the $\rho$ one, that is

$$
t \approx t_{f}+\left(\frac{d t}{d P}\right)_{f}\left(P-P_{f}\right)+\cdots+\frac{1}{(\rho-1) !}\left(\frac{d^{\rho-1} t}{d P^{\rho-1}}\right)_{f}\left(P-P_{f}\right)^{\rho-1}+\frac{1}{\rho !}\left(\frac{d^{\rho} t}{d P^{\rho}}\right)_{f}\left(P-P_{f}\right)^{\rho} .
$$

Because $\frac{d^{\lambda} t}{d P^{\lambda}}=0(\lambda<\rho)$ at $t_{f}$, we get

$$
P \approx P_{f}-\left[-\frac{1}{\rho !}\left(\frac{d^{\rho} t}{d P^{\rho}}\right)_{f}\right]^{-\frac{1}{\rho}}\left(t_{f}-t\right)^{\frac{1}{\rho}}
$$

And thus

$$
\frac{d P}{d t} \approx \frac{1}{\rho}\left[-\frac{1}{\rho !}\left(\frac{d^{\rho} t}{d P^{\rho}}\right)_{f}\right]^{-\frac{1}{\rho}}\left(t_{f}-t\right)^{-\left(1-\frac{1}{\rho}\right)} .
$$

So the break rate has a critical power law relation

$$
\frac{d P}{d t} \propto\left(t_{f}-t\right)^{-\beta} \text {. }
$$


with respect to the time-to-failure with an exponent

$$
-\beta=-\left(1-\frac{1}{\rho}\right) .
$$

Thus, $-\beta$ ranges from $-1 / 2$ to -1 when $\rho$ ranges from 2 to infinity. Lennartz-Sassinek et al. [37] define a relationship $\beta=-\gamma /(\gamma+1)$ of the critical exponent $\beta$ with the exponent $\gamma$ that describes the time-dependent weakening of local strength. Different from the present model that describes a non-linear dependence of lifetime of each fiber on the history of its local stress, this prior model [37] assumes a time-dependent nonlinear weakening of local strength, which in turn controls the inter-event time.

In fact, accelerating failure could occur for all values of the exponent $\rho>1$, thus $\beta$ ranges from 0 to 1.0 [13]. When $\rho$ is less than 2, Eq. (4) leads to $(d t / d P)_{f}=0$ and $\left(d^{2} t / d P^{2}\right)_{f}$ is infinite at the failure time. As a consequence, the curve has infinite curvature at the failure point. Thus, the transition of the values of $\rho$ to $\rho<2(\beta<1 / 2)$ could represent a transition between two different failure modes.

It has been noted [34] that the subcritical crack growth rate model could correspond to the power-law breakdown model of Eq. (8) for fiber elements. Then Charles' [38] power law

$$
V=V_{0} \exp (-h / R T) K^{\rho} \text {. }
$$

that describes subcritical crack growth [36] can be analogous to the breakdown rule (Eq. (8)) of a single fiber, where $V$ is the crack velocity, $K$ is the stress intensity factor, $h$ is the activation enthalpy, $R$ is the gas constant and $T$ is the absolute temperature. Values of the exponent $\rho$ have been experimentally observed to range from 9.5 to as large as 170 for some rocks ruptured under tension [34,36], so that the value of the critical exponent $-\beta$ is close to -1.0 . This has a significant implication that the failure time can be predicted by linearly extrapolating the curve of the inverse rate to zero [1-4].

\subsection{Numerical results under ELS}

We performed Monte Carlo simulations of the breaking process of the time-dependent fiber-bundle model under ELS to explicitly show the magnitude and variation of critical power law exponents. First, random lifetimes are generated according to the Weibull distribution of Eq. (9). The first break occurs at the fiber with the shortest lifetime among the distribution of generated lifetimes. The consumed lifetime in this step of every surviving fiber is calculated from Eq. (7). Then, the fiber with the minimum remaining lifetime is then found and scrutinized to define its failure. The load concentration factor is calculated from Eq. (6), and the actual loads on all surviving fibers are recalculated according to Eq. (4). This process continues until the fiber bundle has totally failed.

The break-rate of the bundle in the numerical simulations is defined as $\zeta(t)=\frac{d P}{d t}$. Fig. 1 illustrates the evolution with time of the number of broken fibers $N_{b}$ for the cases with different values of $\rho$. It is clear that higher value of $\rho$ leads to a sharper increase in the number of broken fibers nearby the failure time $t_{f}$. As examples, Fig. 2 shows the log-log plots of the break rate $\zeta(t)$ with time to failure in the vicinity of failure for $\rho=2.0,2.5,3.0,5.0$ and $\rho=10$. It is shown that the general trend of the breaking rate $\zeta(t)$ agrees well with the critical power law relation, Eq. (18) in the vicinity of failure time. In practical application, the inconsistency of the error structure of the rate data after the transformation [23] could lead to bias in the regression fits of the exponent $\beta$.

The linear least squares fit to the data of the log-log curves (Fig. 2) shows that the critical exponent $-\beta=-0.50$ for $\rho=2.0,-\beta=-0.60$ for $\rho=2.5,-\beta=-0.67$ for $\rho=3.0,-\beta=-0.80$ for $\rho=5.0$, and $-\beta=-0.90$ for $\rho=10.0$. Fig. 3 shows that the evolution of the simulated results of $\beta$ with a change in $\rho$ under ELS, which illustrates that $\beta$ has a relationship with $\rho$ as $\beta=1-1 / \rho$. This agrees well with the analytical derivation.

\section{Summary and conclusions}

Based on the ELS rule and the series expansion in the vicinity of failure time, we find that the critical power law exponent $-\beta$ ranges from -0.5 to -1.0 . The curve has an infinite curvature at the failure point when $\beta<1 / 2$, which could represent the transition to a different failure mode. The variation of the critical power law exponents observed in field and laboratory data has its intrinsic physical controls, but not only due to the fluctuation in the measurement data. The parameter $\rho$ that defines the dependence of the damage of a fiber on the local stress, has an important influence on the critical power law exponent. The simulation results show that the critical power law exponent $-\beta$ has a relationship with $\rho$ that $-\beta=-\left(1-\frac{1}{\rho}\right)$. This is confirmed by an analytical derivation based on a continuous model. Thus, in time-dependent failure, the value of the critical exponent of the power law precursor is determined by the degree to which the local stress controls the damage evolution.

\section{Acknowledgments}

This work is supported by National Natural Science Foundation of China (Grant no. 11672258), National Basic Research Program of China (Grant no. 2013CB834100) and Natural Science Foundation of Hebei Province, China (Grant D2015203398). 


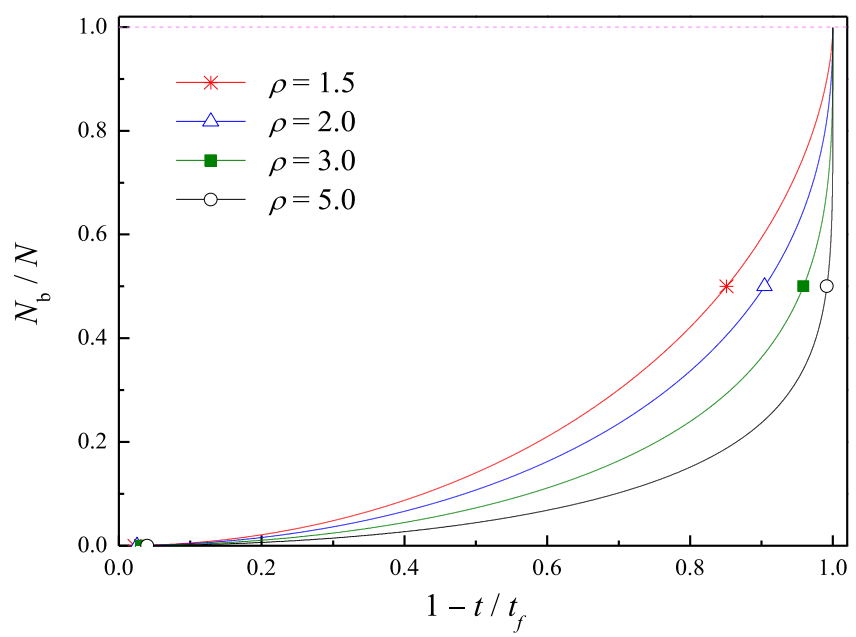

Fig. 1. Evolution of the number of broken fibers versus time for different $\rho$ in the ELS model. The results were obtained by Monte Carlo simulation with $\theta=2, \sigma_{0} / \eta=1.0, \kappa_{0}=1.0$ and a total number of fibers $\mathrm{N}=30,000$.

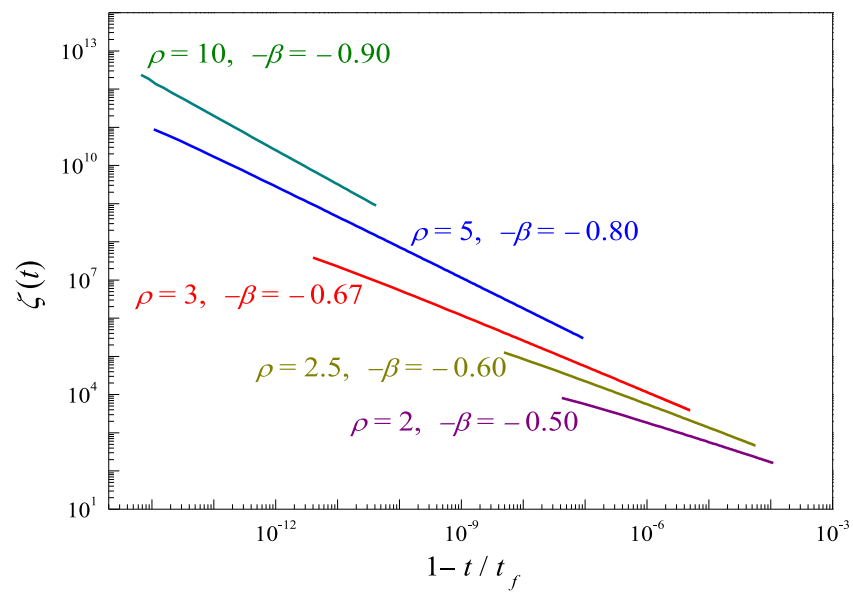

Fig. 2. Critical power law behavior for different $\rho$ in the ELS model. Monte Carlo simulation with $\theta=2, \sigma_{0} / \eta=1.0, \kappa_{0}=1.0$ for total number of fibers $\mathrm{N}$ $=30,000$.

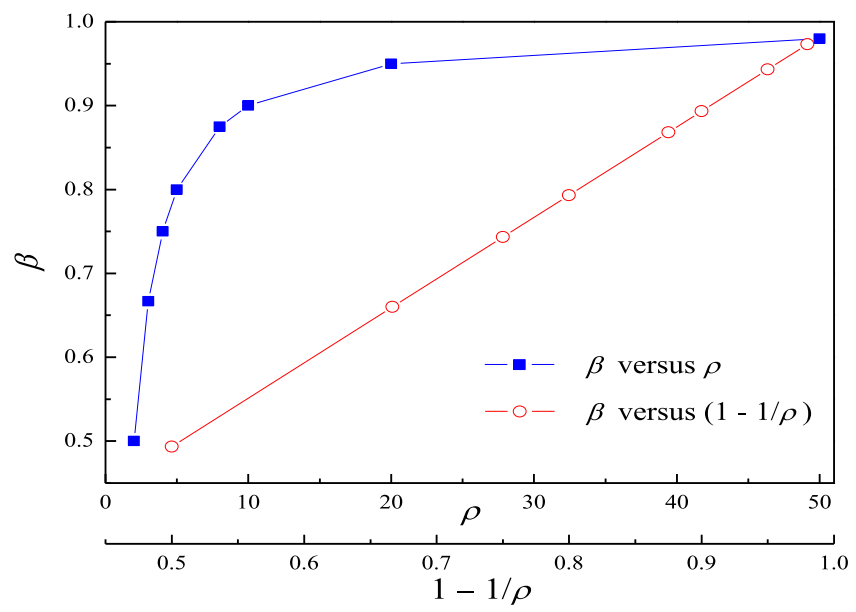

Fig. 3. Curves of $\beta$ versus $\rho$ and $(1-1 / \rho)$. 


\section{References}

[1] B. Voight, A relation to describe rate-dependent material failure, Science 243 (1989) 200-203.

[2] B. Voight, A method for prediction of volcanic eruptions, Nature 332 (1988) 125-130.

[3] B. Voight, R.R. Cornelius, Prospects for eruption prediction in near real-time, Nature 350 (1991) $695-698$.

[4] C.R.J. Kilburn, D.N. Petley, Forecasting giant catastrophic slope collapse: Lessons from Vajont, northern Italy, Geomorphology 54 (1-2) (2003) 21-32.

[5] C.R.J. Kilburn, B. Voight, Slow rock fracture as eruption precursor at Soufriere Hills, volcano, Montserrat, Geophys. Res. Lett. 25 (19) (1998) 3665-3668.

[6] C.R.J. Kilburn, Precursory deformation and fracture before brittle rock failure and potential application to volcanic unrest, J. Geophys. Res. 117 (2012) B02211. http://dx.doi.org/10.1029/2011JB008703.

[7] I.G. Main, Applicability of time-to-failure analysis to accelerated strain before earthquakes and volcanic eruptions, Geophys. J. Int. 139 (1999) F1-F6.

[8] S.W. Hao, C. Liu, C.S. Lu, D. Elsworth, A relation to predict the failure of materials and potential application to volcanic eruptions and landslides, Sci. Rep. 6 (2016) 27877. http://dx.doi.org/10.1038/srep27877.

[9] R. Toussaint, S.R. Pride, Fracture of disordered solids in compression as a critical phenomenon. I. Statistical mechanics formalism, Phys. Rev. E 66 (3) (2002) 036135. http://dx.doi.org/10.1103/PhysRevE.66.036135.

[10] R. Toussaint, S.R. Pride, Fracture of disordered solids in compression as a critical phenomenon. II. Model Hamiltonian for a population of interacting cracks, Phys. Rev. E 66 (3) (2002) 036136. http://dx.doi.org/10.1103/PhysRevE.66.036136.

[11] R. Toussaint, S.R. Pride, Fracture of disordered solids in compression as a critical phenomenon. III. Analysis of the localization transition, Phys. Rev. E 66 (3) (2002) 036137. http://dx.doi.org/10.1103/PhysRevE.66.036137.

[12] R. Toussaint, S.R. Pride, Interacting damage models mapped onto ising and percolation models, Phys. Rev. E 71 (4) (2005) 046127. http://dx.doi.org/ 10.1103/PhysRevE.71.046127.

[13] I.G. Main, A damage mechanics model for power-law creep and earthquake aftershock and foreshock sequences, Geophys. J. Int. 142 (2000) 151-161.

[14] A.F. Bell, M. Naylor, M.J. Heap, I.G. Main, Forecasting volcanic eruptions and other material failure phenomena: An evaluation of the failure forecast method, Geophys. Res. Lett. 38 (15) (2011) L15304.

[15] R. Cornelius, B. Voight, Seismological aspects of the 1989-1990 eruption at Redoubt Volcano, Alaska: The Materials Failure Forecast Method (FFM) with RSAM and SSAM seismic data, J. Volcanol. Geotherm. Res. 62 (1994) 469-498.

[16] R. Cornelius, B. Voight, Graphical and PC-software analysis of volcano eruption precursors according to The Materials Failure Forecast Method (FFM), J. Volcanol. Geotherm. Res. 64 (1995) 295-320.

[17] A.F. Bell, C.R.J. Kilburn, Precursors to dyke-fed eruptions at basaltic volcanoes: Insights from patterns of volcano-tectonic seismicity at Kilauea volcano, Hawaii, Bull. Volcanol. 74 (2012) 325-339.

[18] Y. Lavallée, et al., Seismogenic lavas and explosive eruption forecasting, Nature 453 (2008) 507-510. http://dx.doi.org/10.1038/nature06980.

[19] R. Smith, P.R. Sammonds, C.R.J. Kilburn, Fracturing of volcanic systems: Experimental insights into pre-eruptive conditions, Earth Planet. Sci. Lett. 280 (2009) 211-219.

[20] A. Helmstetter, D. Sornette, J.R. Grasso, J.V. Andersen, S. Gluzman, V. Pisarenko, Slider-block friction model for landslides: Application to Vaiont and La Clapière landslides, J. Geophys. Res. 109 (2004) B02409. http://dx.doi.org/10.1029/2002JB002160.

[21] D.N. Petley, T. Higuchi, D.J. Petley, M.H. Bulmer, J. Carey, Development of progressive landslide failure in cohesive materials, Geology 33 (3) (2005) 201-204.

[22] R. Smith, C.R.J. Kilburn, Forecasting eruptions after long repose intervals from accelerating rates of rock fracture: The June 1991 eruption of Mount Pinatubo, Philippines, J. Volcanol. Geotherm. Res. 191 (2010) 129-136.

[23] A.F. Bell, M. Naylor, I.G. Main, The limits of predictability of volcanic eruptions from accelerating rates of earthquakes, Geophys. J. Int. 194 (2013) $1541-1553$.

[24] S.W. Hao, H. Yang, D. Elsworth, An accelerating precursor to predict "time-to-failure" in creep and volcanic eruptions, J. Volcanol. Geotherm. Res. 343 (1) (2017) 252-262.

[25] S.W. Hao, B.J. Zhang, J.F. Tian, D. Elsworth, Predicting time-to-failure in rock extrapolated from secondary creep, J. Geophys. Res. 119 (2014) 19421953.

[26] F. Kun, R.C. Hidalgo, H.J. Herrmann, K.F. Pal, Scaling laws of creep rupture of fiber bundles, Phys. Rev. E 67 (2003) 061802.

[27] D.L. Turcotte, W.I. Newman, R. Shcherbakov, Micro and macroscopic models of rock fracture, Geophys. J. Int. 152 (2003) $718-728$.

[28] S.W. Hao, B.J. Zhang, J.F. Tian, Relaxation creep rupture of heterogeneous material under constant strain, Phys. Rev. E 85 (1) (2012) 012501.

[29] H.A. Daniels, The statistical theory of the strength of bundles of threads. I, Proc. R. Soc. London A 183 (1945) $405-435$.

[30] S. Pradhan, A. Hansen, B.K. Chakrabarti, Failure processes in elastic fiber bundles, Rev. Modern Phys. 82 (2010) 499-555.

[31] B.D. Coleman, Statistics and time dependence of mechanical breakdown in fibers, J. Appl. Phys. 29 (6) (1958) 968-983. http://dx.doi.org/10.1063/1. 1723343.

[32] D.G. Harlow, S.L. Phoenix, The chain-of-bundles probability model for the strength of fibrous materials I: Analysis and conjectures, J. Compos. Mater. 12 (2) (1978) 195-214.

[33] D.G. Harlow, S.L. Phoenix, Chain-of-bundles probability model for the strength of fibrous materials. II. A numerical study of convergence, J. Compos. Mater. 12 (3) (1978) 314-334.

[34] W.I. Newman, S.L. Phoenix, Time-dependent fiber bundles with local load sharing, Phys. Rev. E 63 (2001) 021507.

[35] W.A. Curtin, M. Pamel, H. Scher, Time-dependent damage evolution and failure in materials. II. Simulations, Phys. Rev. B 55 (18) (1997) 12051-12061.

[36] B.K. Atkinson, Subcritical crack growth in geological materials, J. Geophys. Res. 89 (1984) 4077-4114.

[37] S. Lennartz-Sassinek, I.G. Main, Z. Danku, F. Kun, Time evolution of damage due to environmentally assisted aging in a fiber bundle model, Phys. Rev. E. 88 (2013) 032802

[38] R. Charles, The static fatigue of glass, J. Appl. Phys. 29 (1958) 1549-1560. 